\title{
THE IMPACT OF AXLE WEIGHT CONSTRAINTS ON THE DEPLOYMENT OF A MIXED-FLEET IN VEHICLE ROUTING DECISIONS
}

\author{
Hanne Pollaris ${ }^{1}$, Gerrit K. Janssens ${ }^{2}$, Kris Braekers ${ }^{3}$, An Caris ${ }^{4}$, Sabine Limbourg ${ }^{5}$ \\ 1,2,3,4 Research Group Logistics, Hasselt University, Hasselt, Belgium \\ ${ }^{5}$ HEC Management School, Université de Liège, Liège, Belgium
}

Received 13 January 2021; accepted 16 March 2021

\begin{abstract}
A vehicle routing problem (VRP), using a mixed fleet of vehicles, with sequencebased pallet loading and axle weight constraints is introduced. The effect of the integration of axle weight constraints in a Fleet Size and Mix VRP is analyzed by comparing the problem with and without axle weight constraints. A vehicle fleet of 30 -foot and 45 -foot trucks, consisting of a tractor and a semi-trailer, is considered. Two scenarios are analyzed with different objective functions. In the first scenario, the objective aims to minimize total distance while in the second scenario the objective aims the minimization of total transport costs. An Iterated Local Search metaheuristic algorithm is used to solve the problem. The results indicate that the impact of axle weight constraints on the solution cost of a vehicle routing problem depends on the fleet composition. Therefore, decisions on the deployment of a mixed-size fleet may be influenced by the integration of axle weight constraints.
\end{abstract}

Keywords: fleet size, mix VRP, axle weight constraints, routing and loading, iterated local search, heterogeneous vehicle fleet.

\section{Introduction}

Vehicle routing makes up an important part of distribution logistics decision-making. Therefore, the vehicle routing problem is a well-studied problem in operations research. The wealth of studies finds its origin both in the relevance of the problem to the practice of distribution and to the complexity of finding (near)-optimal solutions. Both the increase in computing power as the development of efficient algorithms has made that complex variants of the Vehicle Routing Problem (VRP) can be solved in a reasonable time. Therefore, the research is still active, because, nowadays, real-life cases can be handled. The more complex variants with multiple types of constraints are called, in literature, 'rich vehicle VRPs'. There is no strict definition of a Rich VRP: some of them are mentioned in CaceresCruz et al. (2014). As this paper focuses towards practical applications, the following definition is relevant: "non-idealised models that represent the application at hand in an adequate way by including all important optimisation criteria, constraints, and preferences" (Hartl et al., 2006; Hasle and Kloster, 2007). They may include, for example, the introduction of time windows,

${ }^{2}$ Corresponding author: gerrit.janssens@uhasselt.be 
heterogeneous vehicles, incompatibility of products in vehicles, etc. A classification of the main documented Rich VRPs, in terms of constraints, is given in Caceres-Cruz et al. (2014).

Vehicles in the fleet may differ in terms of capacity, costs and other factors such as speed and product compatibility. Besides the routing of vehicles, logistics companies also need to consider the loading of the vehicles. A feasible loading plan is not guaranteed when only total capacity of a vehicle is considered. The loading problem considers efficiency in unloading at the customers' sites, stability of the vehicles, incompatibility of storage in the vehicle with respect to potential damage of the goods, and fulfilment of rules and regulations regarding mass distribution within the vehicle. Pollaris et al. (2015) mention a survey among several Belgian logistics service providers pointing out that they are faced with complex loading problems when planning their route (e.g. multi-dimensional packing constraints, unloading sequence constraints, stability constraints and axle weight limits). Ignorance of these constraints may compromise planning and induce last minute changes resulting in additional costs. Axle weight limits, in particular, impose a challenge for transportation companies since they are faced with high fines when violating the limits. Weigh-In-Motion (WIM) systems on highways monitor axle weight violations of trucks while driving, which increase the probability that axle weight violations are detected (Jacob and Feybell-de La Beaumelle, 2010). Furthermore, trucks with overloaded axles represent a threat for traffic safety and may cause serious damage to the road surface. Since the weight on the axles changes when items are loaded and unloaded, it is important that axle weights are considered during the entire trip of the vehicle and not only at the time the vehicle departs from the depot.

In this paper, a variant of the classical Capacitated VRP is analyzed. The vehicle fleet consists of heterogeneous vehicles and the demand of the customers is defined in terms of numbers of pallets. Sequence-based loading is imposed which ensures that, when arriving at a customer, no pallets belonging to customers, served later on the route, block the removal of the pallets of the current customer. Furthermore, the capacity of a truck is not only expressed in total weight and number of pallets but also in terms of a maximum weight on the axles of the truck.

The goal of this paper is threefold. First, to introduce and solve the Fleet Size and Mix VRP (FSM) with sequence-based pallet loading and axle weight constraints. An unlimited heterogeneous vehicle fleet, sequence-based pallet loading and axle weight limits are considered. To the best of our knowledge, it is the first time that axle weight constraints are incorporated into an FSM. The second goal is to analyze the effect of the integration of axle weight constraints in a VRP for varying vehicle fleet compositions. To this end, three heavy-duty vehicle fleet compositions are compared: a homogeneous fleet of 30-foot trucks, a homogeneous fleet of 45 -foot trucks and a heterogeneous fleet consisting of 30 -foot and 45-foot trucks. The third goal is to analyze the impact of an objective function, which considers the total transport costs on the effect of axle weight constraints in a VRP compared to the more traditional objective of minimizing total distance or travel time.

In the next section, a literature review is presented on the topic. In section 3, 
a problem description of the FSM with sequence-based pallet loading and axle weight constraints is presented. In Section 4, the methodology is described. Section 5 presents an overview of the results. In the final section, conclusions and future research opportunities are presented.

\section{Literature Review}

The literature concerning heterogeneous vehicle fleet routing is divided into two major problem classes (Koç et al., 2014). The first class is called the Fleet Size and Mix Vehicle Routing Problem (FSM) and considers an unlimited vehicle fleet. The problem is introduced by Golden et al. (1984). The objective is to minimize total distribution cost and determine the optimal fleet size and mix. The second problem class is called the Heterogeneous Vehicle Routing problem (HVRP) and considers a limited vehicle fleet. The goal of the HVRP is to minimize total distribution costs, given an available fleet. This problem is introduced by Taillard (1999). Since the introduction of the FSM and HVRP, many solution techniques, mainly heuristics, have been developed. This paper focuses on the FSM. For an overview of the literature, the reader is referred to Koç et al. (2016) and Baldacci et al. (2008).

To our knowledge, Lim et al. (2013) and Alonso et al. (2017) are the only authors that address axle weight constraints in a container loading problem. The integration of axle weight constraints in a Capacitated VRP (CVRP) has been introduced in Pollaris et al. (2016). She solves problems with networks up to 20 nodes by a Mixed Integer Linear Programming model (MILP). In Pollaris et al. (2017) instances with up to 100 customers are solved by an Iterated Local Search (ILS) metaheuristic.
For state-of-the-art reviews of the literature concerning the combination of Vehicle Routing Problems and loading problems, the reader is referred to Iori and Martello (2010) and Pollaris et al. (2015). Since the latter review article, further attention has been paid to loading constraints, for example in Männel and Bortfeldt (2016), Wei et al. (2018) and Paquay et al. (2018). In Männel and Bortfeldt (2016), the Pick-up and Delivery problem (PDP) is extended to an integrated routing three-dimensional loading problem with homogeneous vehicles. Each transportation request is given as a set of 3D boxes. In Wei et al. (2018), a CVRP with two-dimensional loading constraints is studied, involving two-dimensional, rectangular, weighted items. A simulated annealing algorithm is proposed for various versions of the problem, i.e. with or without LIFO constraints, and allowing rotations of the items or not. In Paquay et al. (2018), a three-dimensional multiple bin size bin packing problem is studied, which is typical for air transportation. The problem includes real-life loading constraints regarding fragility, stability and possible orientations of the boxes. A fast heuristic is developed to solve the problem with, additionally, post-processing for weight distribution improvement.

Ostermeier et al. (2018) study the interdependent loading and routing problem in multi-compartment vehicles, in which the size and position of the compartments can be adjusted. Both an exact branch-and-cut algorithm and a large neighbourhood search, with record-to-record travel embedded are developed and investigated. Alonso et al. (2019) study the multi-container problem aiming to load a set of products in a minimum number of containers. Next to the axle weight constraints, as on focus 
in this article, also the distribution of the weight over the truck floor is considered. Additional constraints are considered, which the authors call dynamic stability constraints in order to avoid empty spaces between pallets and to limit excessive differences between the heights of adjacent pallets. Alonso et al. (2020) study dynamic aspects when the vehicle is moving subjected to accelerating and braking forces. They introduce several pallet structures like: stock pallets (homogeneous i.e. containing the same product in all layers), case pallets (homogeneous, but only per layer) and rest pallets (strongly heterogeneous pallets). They develop a GRASP algorithm to solve the problem.

\section{Problem Description}

This paper integrates a heterogeneous vehicle fleet in the CVRP with sequence-based pallet loading and axle weight constraints. As a vehicle fleet is usually heterogeneous in real-life, the extension of the VRP to heterogeneous vehicles is highly relevant (Bräysy et al., 2009).

The assumptions of the problem under study are listed here:

- Customer demand: Demand is expressed as a number of euro pallets $(80 \times 120$ $\mathrm{cm})$. Pallets of a single customer have the same weight and that the weight is uniformly distributed inside each pallet.

- Packing of pallets: Pallets are packed dense in two horizontal rows a left one and a right one), with no gap between two consecutive pallets in the container. Pallets are packed alternating in the left and right row. Dense packing entails that there is an open space allowed between the front of the container and the first pallets that are packed. Dense packing is often imposed to increase the stability of the load since it restricts the moving area of the pallets considerably. The driver therefore needs to spend less time on securing the cargo. Sequence-based loading is imposed. Vertical stacking is not allowed.

- Vehicle types: Vehicles in the fleet are different in terms of tare weight and measurements. Consequently, the capacity in terms of number of pallets and payload is different as well as the weight capacity of the axles.

- Axle weight: Axle weight is defined as the weight that is placed on the axles of the truck. The tractor has two axles: the steering axle and the driving axle, both belong to the tractor. The axles of the trailer are called tridem axles. Tridem axles are three successive axles with a distance of less than 1.8 meter and more than 1 meter between the middle of the first axle and the middle of the second axle, and between the middle of the second axle and the middle of the third axle. The calculation of the division of the weight of an item, placed in the container, over the different axles is developed in Pollaris et al. (2016).

In Europe, heavy goods vehicles, buses and coaches must comply with certain rules on weights and dimensions for road safety reasons and to avoid damaging roads, bridges and tunnels. A directive of the European Union (EU) has set maximum dimensions and weights for international traffic, also ensuring that Member States cannot restrict the circulation of vehicles which comply with these limits for performing international transport operations within their territories. Another directive grants derogations on the maximal lengths to make heavy goods vehicles greener by improving their aerodynamic performance. These rules 
are complemented by the requirements for type-approval of commercial vehicles laid out in Directive 97/27/EC and its implementing measures. These legal acts set the framework for putting vehicles such as light-duty and heavy-duty vehicles, buses and trailers on the market. In this article, the Belgian legislation is taken as an example. Belgian legislation (KB 15.03.1968 art 32 bis) incorporated European Directive 97/27/EC that specifies that the mass corresponding to the load on the driving axle must be at least 25 percent of the total mass of the loaded truck. There are no specific guidelines concerning the lower bound on the weight on the axles of the trailer, except for the fact that it can never be negative since a negative weight on one of the axles would cause the truck to overturn.

\subsection{Model Formulation: Objective Function, Constraints and Notation}

Traditionally, in literature, the minimization of the total distance traveled is chosen as an objective. However, in this case, the objective function aims to minimize total transport costs. In such a way, a distinction can be made between hour costs and distance (say kilometer) costs. This objective corresponds more to the objective of transportation companies in real-life. It is investigated whether the choice of objective influences the results of the analysis. Intuitively, it may be expected that the impact of the integration of axle weight constraints in scheduling of routes is smaller using the second objective. Using this second objective, fuel costs are taken into consideration, which depend on the gross weight of the vehicle. Routes visiting customers with the heaviest pallets early in the route therefore will be favored over routes in which these customers are visited further in the route, due to the effect in order to minimize fuel costs. As sequencebased loading is assumed, this implies that heavy pallets are placed towards the rear of the vehicle, carried by the axles of the trailer. Since the axles of the trailer have a larger weight capacity, this leads to a smaller probability of an axle weight violation. The formulation of the model is partially inspired by Hiermann et al. (2016).

Before the formulation of the optimization model, the notations are introduced. An instance of the problem under study consist of a set of customers $V$, a depot node and $k$ different vehicle types.

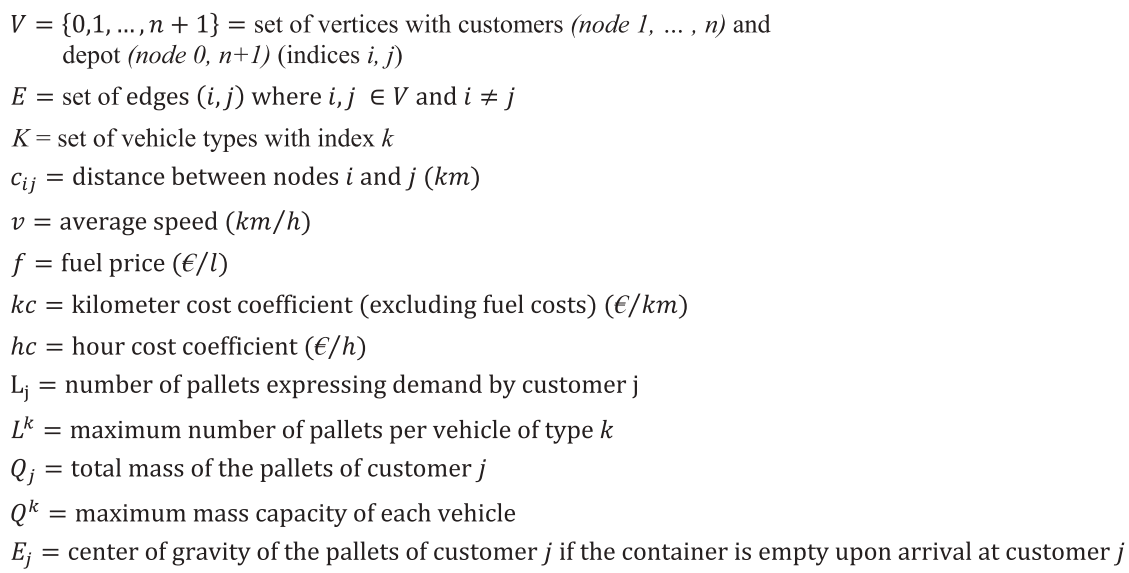


$O_{j}=$ center of gravity of the pallets of customer $j$ if 1 pallet is inthe container upon arrival at customer $j$

$A^{k F}=$ maximum weight on the coupling for a vehicle of type $k$

$A^{k R}=$ maximum weight ont he axles of the semi - trailer for a vehicle of type $k$

$W^{k T}=$ mass of the empty vehicle of type $k$

$W^{k T D}=$ weight of the empty vehicle of type $k$ on the driving axle

$W^{k T R}=$ weight of the empty vehicle of type $k$ on the axles of the semi - trailer

$h=$ fraction of the weight ont he coupling that is carried by the driving axle

$c^{k}=$ distance between the front of the container and the coupling for a vehicle of type $k$

$d^{k}=$ distance between the coupling and the center of the axles of the semi - trailer

$P_{j}=1$ if $L_{j}$ is even, -1 if $L_{j}$ is odd

\section{The decision variables are defined as follows:}

$x_{i j}^{k}=\left\{\begin{array}{cc}1 & \text { if a vehicle of type } k \text { travels from } i \text { to } j \text { with }(i, j) \in E \\ 0 & \text { otherwise }\end{array}\right.$

$f e_{i j}=$ fuel efficiency on $(i, j)$ considering the gross weight of the truck on $(i, j)(\mathrm{km} / \mathrm{l})$

$l_{i j}^{k}=\left\{\begin{array}{c}\text { total number of pallets on }(i, j), \quad \text { if a vehicle of type } k \text { travels from } i \text { to } j \text { with }(i, j) \in E \\ 0\end{array}\right.$

$q_{i j}^{k}=\left\{\begin{array}{cc}\text { total cargo mass on }(i, j), & \text { if a vehicle of type } k \text { travels from } i \text { to } j \text { with }(i, j) \in E \\ 0 & \text { otherwise }\end{array}\right.$

$a_{i j}^{k F}=\{$ total cargo weight on the coupling on $(i, j), \quad$ if a vehicle of type $k$ travels from $i$ to $j$ with $(i, j) \in E$ 0 otherwise

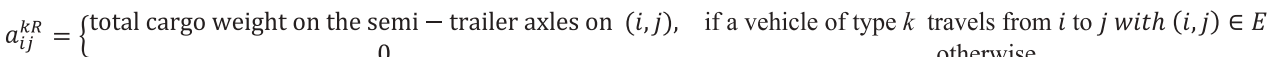
$C G_{j}=$ center of gravity of the pallets of customer $j$ $C_{i j}^{k}=\left\{\begin{array}{c}1 \text { if } l_{i j}^{k} \text { is even (or }-1 \text { if } l_{i j}^{k} \text { is odd) and a vehicle travels of type } k \text { from } i \text { to } j \text { with }(i, j) \in E \\ 0 \text { otherwise }\end{array}\right.$

The (non-linear) objective function may be $\quad \sum_{k \in K} x_{j, 0}^{k}=0, \forall j \in V$ formulated as follows:

$$
\sum_{k \in K} l_{0 j}^{k}=0, \forall j \in V
$$

$\operatorname{Min} \sum_{(i, j) \in E}\left(k c+\frac{h c}{v}+\frac{1}{f e_{i j}^{k}} \cdot f\right) \cdot c_{i j} x_{i j}^{k}$

$l_{i j}^{k} \leq L^{k} x_{i j}^{k}, \forall(i, j) \in E$ and $i \neq j, \forall k \in K$

Subject to the constraints:

$\sum_{i \in V, i \neq j}\left(l_{i j}^{k}+L_{j} x_{i j}^{k}\right)=\sum_{p \in V, p \neq j} l_{j p}^{k}+, \forall j \in V \backslash\{0, n+1\}$

$$
\begin{array}{lll}
\sum_{k \in K} \sum_{i \in V, i \neq j} x_{i j}^{k}=1, \forall j \in V \backslash\{0, n+1\} & \text { (2) } & \sum_{k \in K} q_{0 j}^{k}=0, \forall j \in V \\
\sum_{k \in K} \sum_{j \in V, j \neq i} x_{i j}^{k}=1, \forall i \in V \backslash\{0, n+1\} & \text { (3) } q_{i j}^{k} \leq Q^{k} x_{i j}^{k}, \forall(i, j) \in E \text { and } i \neq j, \forall k \in K \\
\sum_{i \in V \backslash\{0\}, i \neq j} x_{j i}^{k}-\sum_{i \in V \backslash\{n+1\}, i \neq j} x_{i j}^{k}=0, \forall j \in V\{0, n+1\} & \text { (4) } \sum_{i \in V, i \neq j} q_{i j}^{k}+Q_{j} x_{i j}^{k}=\sum_{p \in V, p \neq j} q_{j p,}^{k}, \forall j \in V \backslash\{0, n+1\} \\
\sum_{k \in K} x_{n+1, j}^{k}=0, \forall j \in V & \text { (5) } C_{0 j}^{k}=x_{0 j}^{k}, \forall j \in V, \forall k \in K
\end{array}
$$




$$
\begin{aligned}
& C_{i j}^{k} \leq x_{i j}^{k}, \forall(i, j) \in E \text { and } i \neq j, \forall k \in K \\
& C_{i j}^{k} \geq-x_{i j}^{k}, \forall(i, j) \in \text { Eand } i \neq j, \forall k \in K \\
& \sum_{k \in K} \sum_{i \in V, i \neq j} C_{i j}^{k} P_{j}=\sum_{k \in K} \sum_{p \in V, p \neq j} C_{j p}^{k}=, \forall j \in V \backslash\{0, n+1\} \\
& a_{0 J}^{k F}=0, \forall j \in V, \forall k \in K \\
& a_{0 J}^{k R}=0, \forall j \in V, \forall k \in K \\
& a_{i j}^{k F} \leq A^{k F} x_{i j}^{k}, \forall(i, j) \in E \text { and } i \neq j, \forall k \in K \\
& a_{i j}^{k R} \leq A^{k R} x_{i j}^{k}, \forall(i, j) \in E \text { and } i \neq j, \forall k \in K \\
& a_{i j}^{k F} \geq-W^{k T D} x_{i j}^{k}, \forall(i, j) \in E \text { and } i \neq j, \forall k \in K \\
& a_{i j}^{k R} \geq-W^{k T R} x_{i j}^{k}, \forall(i, j) \in E \text { and } i \neq j, \forall k \in K \\
& a_{i j}^{k F} h+W^{T D} \geq 0.25\left(W^{T}+q_{i j}^{k}\right), \forall(i, j) \in E \text { and } i \neq j, \forall k \in K \\
& \sum_{i \in V, i \neq j} a_{i j}^{k F}+Q_{j} x_{i j}^{k}-\frac{\left(c G_{j}^{k}-c^{k}\right) Q_{j} x_{i j}^{k}}{d^{k}}=\sum_{p \in V, p \neq j} a_{j p}^{k F}, \forall j \in V, \forall k \in K \\
& \sum_{i \in V, i \neq j} a_{i j}^{k R}-\frac{\left(c G_{j}^{k}-c^{k}\right) Q_{j} x_{i j}^{k}}{d^{k}}=\sum_{p \in V, p \neq j} a_{j p}^{k R}, \forall j \in V, \forall k \in K \\
& C G_{j}^{k}=\frac{\sum_{i \in V, i \neq j} l_{i j}^{k}}{2}-\frac{1}{4}\left(1-\sum_{i \in V, i \neq j} C_{i j}^{k}\right)+\frac{1}{2} O_{j}^{k}(1- \\
& \left.-\sum_{i \in V, i \neq j} C_{i j}^{k}\right)+\frac{1}{2} E_{j}^{k}\left(1+\sum_{i \in V, i \neq j} C_{i j}^{k}\right), \forall j \in V, \forall k \in K \\
& x_{i j}^{k} \in[0,1], \forall(i, j) \in E \text { and } i \neq j \\
& l_{i j}^{k} \geq 0, \forall(i, j) \in E \text { and } i \neq j \\
& q_{i j}^{k} \geq 0, \forall(i, j) \in E \text { and } i \neq j
\end{aligned}
$$

The objective function (1) aims to minimize the total transport costs.

Constraints (2) and (3) ensure that each customer is visited exactly once. Constraint (4) states that, in case a vehicle of type $k$ arrives at a customer, also a vehicle of type $k$ has to leave the customer. Constraint (5) makes sure that no route starts at the final depot (node $n+1)$, while constraint (6) ensures that no route arrives in the start depot (node 0). Constraints (7) and (10) initialize the values of $l_{0 j}{ }^{k}$ and $q_{0 j}{ }^{k}$ to 0 , since a container is empty when it leaves the start depot. Constraint (8) limits $l_{i j}^{k}$ to the maximum number of pallets that may be placed in a vehicle of type $k$. Constraint (9) keeps track of $l_{i j}{ }^{k}$ by adding up the number of pallets when arriving at customer $j\left(l_{i j}{ }^{k}\right)$ with the number of pallets of customer $j$ $\left(L_{j}\right)$. Constraint (12) keeps track of $q_{i j}{ }^{k}$ in a similar way. Note that dense packing of the pallets into the vehicle is imposed, i.e. no gap between two consecutive pallets in the truck is allowed. Constraint (11) limits $q_{i j}{ }^{k}$ to the maximum mass capacity $\left(Q^{k}\right)$ of the vehicle. In constraint (13), the value of the variable $C_{0 j}{ }^{k}$ is set to 0 if $x_{0 j}{ }^{k}=0$ and set to 1 if $x_{0 j}{ }^{k}=1$. Since a container is empty when it departs from the start depot, it has an even number of pallets ( 0 pallets). Constraints (14) and (15) guarantee that $C_{i j}{ }^{k}$ can only have a non-zero value in case a vehicle travels from $i$ to $j$. Constraint (16) keeps track of $C_{i j}{ }^{k}$ by multiplying the value of $C_{i j}{ }^{k}$ when arriving at customer $j$ with parameter $P_{j}$. Constraints (17) and (18) initialize the value of the weight on the coupling $\left(a_{i j}{ }^{k F}\right)$ and the weight on the rear axles $\left(a_{i j}{ }^{k R}\right)$ to zero. Constraints (19) till (22) ensure that $a_{i j}{ }^{k F}$ and $a_{i j}{ }^{k R}$ only have a non-zero valuein case a vehicle of type $k$ travels from $i$ to $j$. The values of the upper bounds $A^{k F}$ and $A^{k R}$ depend on the vehicle characteristics and are specified by legislation. For example, Belgian legislation (KB 15.03.1968 art 32bis) specifies that the mass corresponding to the load on the driving axle must be at least 25 percent of the total mass of the loaded truck. This regulation is captured in constraint (23). On the left-hand side of constraint (23), the weight of the empty vehicle on the driving axle $\left(W^{T D}\right)$ is added to the weight of the load that is placed on the coupling $\left(a_{i j}{ }^{k F}\right)$ 
multiplied by a parameter $h$ (percentage of the weight of the load on the coupling, that is carried by the driving axle of the tractor). Since there exist no guidelines concerning the lower bound of the weight on the axles of the semi-trailer, constraint (22) ensures that this would be at least $-W^{T R}$ to avoid a negative axle weight on the rear axles. Constraint (24) keeps track of $a_{i j}{ }^{k F}$ by adding the weight on the coupling when arriving at customer $j\left(a_{i j}{ }^{k F}\right)$ to the weight on the coupling of the pallets of customer $j$. Constraint (25) keeps track of $a_{i j}{ }^{k R}$ in a similar way. Constraint (26) determines the center of gravity of the pallets of customer $j\left(\mathrm{CG}_{\mathrm{j}}\right)$ as a function of $C_{i j}{ }^{k}$ and $l_{i j}{ }^{k}$. A detailed explanation of how constraint (26) is obtained, is given in Appendix A.

Regarding the objective function, the assumption is made that the cost figures are equal for the different vehicle types. Contacts with a logistics service provider confirm that purchasing costs as well as maintenance costs are comparable for heavy-duty vehicles with the same axle configuration (tractor and semi-trailer with tridem axles). The hour cost coefficient $h c$ consists of fixed costs that are charged per hour. The hour cost coefficient is divided by the average speed of a vehicle to calculate the cost per kilometer. In the kilometer cost coefficient $k c$ the fuel cost is excluded. Fuel consumption depends on the gross weight of the truck. The objective function is therefore not linear. The effect of weight on the fuel efficiency of heavy-duty vehicles can be characterized by a linear function $f e_{i j}^{k}=\pi+\tau \cdot L^{k}$ (Kopfer et al. (2014); Xiao et al. (2012)). This formula presents the fuel efficiency in function of the weight of the payload $L^{k}$. For the values of $\pi$ and $\tau$, a study on the fuel efficiency of heavy-duty vehicles of the UK Department of Transport (2007) is consulted. Since the tare weight of the vehicles in a heterogeneous fleet may be different, fuel efficiency depends on the vehicle type.

Note that only weight is included in the calculation of the fuel efficiency, while other factors such as road gradient, speed and acceleration are not considered. It is assumed that these factors are constant for the different vehicle types since the vehicles in the heterogeneous fleet are assumed to be heavy-duty vehicles. In case a mix between low-duty, medium-duty and heavy-duty vehicles with different values for average speed and acceleration are considered in the vehicle fleet, fuel efficiency models that include these factors may be used. For an overview of fuel consumption models in literature, the reader is referred to Kopfer et al. (2014) and Demir et al. (2011).

\section{Methodology}

The effect of the integration of axle weight constraints in an FSM is analyzed by comparing the FSM with and without axle weight constraints. A vehicle fleet of 30 -foot and 45-foot trucks, consisting of a tractor and a semi-trailer, is considered. Furthermore, the impact of the vehicle fleet on the integration of axle weight constraints in a VRP is measured by comparing a heterogeneous fleet with 30 -foot and 45 -foot trucks to a homogeneous fleet with 30-foot trucks and a homogeneous fleet with 45 -foot trucks. The characteristics of both vehicle types (measurements, capacity, mass, axle weight limits) are based on information from a Belgian logistics service provider. In Table 1 , the main characteristics of the 30 -foot and 45 -foot truck are summarized. Note that the total weight capacity as well as the weight capacity of the axles is smaller for the 45-foot truck than for the 30-foot truck. The reason for this is that the tare weight of 
a 45 -foot truck (14.65 ton) is higher than the tare weight of a 30 -foot truck (11.8 ton), while the limits on the gross weight (44 ton) and on the axles of the loaded truck remain unchanged. An unlimited number of both
30 -foot and 45-foot vehicles is considered. The price of fuel ( $f$ in equation (1)) is fixed to $€ 0.70$ per liter. For the average speed of a truck ( $v$ in equation (1)) a value of $50 \mathrm{~km} / \mathrm{h}$ is adopted.

\section{Table 1}

Comparison of the Characteristics of the 30-foot Truck and 45-foot Truck

\begin{tabular}{|l|c|c|}
\hline & $\mathbf{3 0}$-foot & $\mathbf{4 5}$-foot \\
\hline Tare Weight & $11.8 \mathrm{t}$ & $14.65 \mathrm{t}$ \\
\hline $\begin{array}{l}\text { Capacity in Terms } \\
\text { of Pallets }\end{array}$ & 22 & 32 \\
\hline Weight Capacity & $32.2 \mathrm{t}$ & $29.35 \mathrm{t}$ \\
\hline Max Weight Coupling & $11.6 \mathrm{t}$ & $10.75 \mathrm{t}$ \\
\hline $\begin{array}{l}\text { Max Weight } \\
\text { Axles Trailer }\end{array}$ & $21 \mathrm{t}$ & $18.84 \mathrm{t}$ \\
\hline
\end{tabular}

For the computational tests, instances with networks consisting of 50,75 and 100 customers are generated. The instances are created with randomly generated customer location coordinates $(x, y)$ with $x \in[0,250]$ and $y \in[0,250]$. The position of the depot is fixed to the coordinate $(125,125)$. Routing costs are computed by taking the Euclidean distance between the coordinates of each node pair. Four problem classes are defined based on the number of pallets per customer and the weight of the demand. The problem classes are presented in Table 2. Preliminary analysis points out that, in case only heavy pallets $(1000-1500 \mathrm{~kg}$ ) are considered, a feasible loading plan cannot be achieved in a 45-foot truck. When more than 11 pallets of $1000 \mathrm{~kg}$ are packed dense in the truck, the weight on the coupling exceeds the limit of
10.75 ton. For this reason, problem classes 1 and 2 , in which only heavy pallets are considered, are not included in the instance set. Problem classes 3 and 4, in which a fifty-fifty percent mix between customer demands with light pallets $(100-500 \mathrm{~kg})$ and customer demands with heavy pallets are considered, are included in the analysis. The number of pallets has a low variation (between 4 and 7 pallets per customer) in problem class 3 and a high variation (between 1 and 15 pallets per customer) in problem class 4 . The number of pallets and the total weight for each customer are generated randomly in the intervals above mentioned, depending on the problem class. For each network size, eight instances are created in each problem class, leading to a total of 48 test instances.

\section{Table 2}

Problem Classes based on Variation in Number of Pallets and Pallet Weight

\begin{tabular}{|l|c|c|}
\hline & Heavy Pallets & $\begin{array}{c}\text { Mix between Light and } \\
\text { Heavy Pallets }\end{array}$ \\
\hline Low Variation & Problem Class 1 & Problem Class 3 \\
\hline High Variation & Problem Class 2 & Problem Class 4 \\
\hline
\end{tabular}


The proposed solution method is based on an Iterated Local Search (ILS) framework which is proven to be a highly effective heuristic for routing problems (Lourenço et al., 2010). The ILS consists of four procedures (Generate initial solution, Local Search, Perturbation, Acceptance Criterion). First, an initial solution is constructed. This solution is improved using local search until a local optimum is reached. The local search is performed by a Variable Neighborhood Descent (VND) method. A new starting point for the local search is generated by perturbing the current solution. The acceptance criterion determines, after the local search, with which solution the process continues. The ILS stops after a specified number of consecutive non-improving iterations. A non-improving iteration is an iteration in which no new best solution is found.

The method has been applied successfully by Pollaris et al. (2017) for a situation, in which homogeneous vehicles are used. In a first scenario, the minimization of total distance traveled is used as the objective function. The algorithm is needs to be adapted only regarding the loading feasibility check, because the different vehicle types do not have the same characteristics in terms of capacity. In case a given route does not lead to a feasible packing plan for the first vehicle type, the feasibility is checked for the other vehicle type. As an unlimited number of vehicles is considered and the objective function value does not depend on the vehicle type, it does not matter which vehicle type is used when both vehicle types lead to a feasible packing. In a second scenario, the objective function aims to minimize the total transport costs. For this scenario, vehicle dependent routing costs are considered since the tare weight of the truck is included in the calculation of the fuel consumption. In case both vehicle types lead to a feasible packing plan for a given route, the lightest vehicle is chosen as this choice leads to the lowest transport costs.

The experiments are run on a Xeon E5$2680 \mathrm{v} 3 \mathrm{CPU}$ at $2.5 \mathrm{GHz}$ with $64 \mathrm{~GB}$ of RAM. Because of the stochastic character of the ILS algorithm, ten independent runs of the algorithm are performed and average results are reported.

\section{Experimental Results}

In this section, the effect of a heterogeneous vehicle fleet on the integration of axle weight constraints in a CVRP is analysed. The Fleet Size and Mix VRP with sequence-based pallet loading and axle weight constraints with 30-foot and 45-foot trucks is compared to a homogeneous vehicle fleet CVRP with 30 -foot trucks and a homogeneous fleet CVRP with 45 -foot trucks. Section 5.1 describes the results for the three fleet compositions with the objective to minimize total distance (scenario 1). In Section 5.2, the results for the objective to minimize total transport costs are described (scenario 2).

\subsection{Results for the Objective 'Distance Minimization'}

In this section, the results of the first scenario are discussed. The first scenario makes use of the objective function minimizing total distance. Using the notation, presented in section 3 , the objective function is formulated as:

$\min \sum_{(i, j) \in E, i \neq j} c_{i j} x_{i j}$

Table 3 gives an overview of the percentage of routes in the final solutions of the FSM with 
and without axle weight constraints, under the first scenario, that may be performed by 30 -foot trucks, 45 -foot trucks or by both types of trucks. The majority of the routes can only be performed by 45 -foot trucks. There is, however, a difference between the FSM with and without axle weight constraints.

In the model without axle weight constraints $93.43 \%$ of the routes in the final solution can only be performed by 45 -foot trucks and only $0.34 \%$ can only be performed by 30 -foot trucks. The remaining part may be performed by both types of trucks. This indicates that in the largest part of the routes more than 22 pallets (the capacity of a 30 -foot truck) are transported. The small percentage of routes that can only be performed by 30 foot trucks indicates that the total weight of the load that is transported on these routes is rarely higher than 29.35 tonnes (the weight capacity of a 45 -foot truck).
In the model with axle weight constraints the percentage of routes that can only be performed by 45 -foot trucks drops to 85.10 $\%$. The number of routes that can only be performed by 30 -foot trucks increases to $14.44 \%$, which is considerably higher than in the model without axle weight constraints. The explanation for the shift of 45 -foot trucks to 30 -foot trucks in the model with axle weight constraints is twofold. First, the tare weight of a 45-foot truck is higher than that of a 30-foot truck, so the maximum weight of the load that may be applied on the axles of a 45-foot truck is lower. Second, because the distances between the front of the truck and the coupling and between the coupling and the central axle of the semitrailer are larger in 45-foot trucks, more weight is placed on the coupling. While, for 30-foot trucks, already most violations occur on the coupling in the model without axle weight constraints, a violation on the coupling for 45 -foot trucks is more probable.

\section{Table 3}

Routes Performed by 30-foot Trucks and 45-foot Trucks in the Final Solutions of the FSM in Percentage - Scenario 1

\begin{tabular}{|l|c|c|c|}
\hline & 30 ft Truck & $\mathbf{4 5} \mathbf{f t}$ Truck & $\begin{array}{c}\text { 30 ft or } \\
\mathbf{4 5} \mathbf{f t} \text { Truck }\end{array}$ \\
\hline $\begin{array}{l}\text { FSM without Axle } \\
\text { Weight Constraints }\end{array}$ & $0.34 \%$ & $93.43 \%$ & $6.23 \%$ \\
\hline $\begin{array}{l}\text { FSM with Axle } \\
\text { Weight Constraints }\end{array}$ & $14.44 \%$ & $85.10 \%$ & $0.46 \%$ \\
\hline
\end{tabular}

Table 4 presents the relative decrease in total distance travelled in the solution of the CVRP with sequence-based pallet loading and axle weight constraints, both with a homogeneous vehicle fleet consisting of 45 -foot trucks and with a heterogeneous vehicle fleet with 30 -foot and 45 -foot trucks, compared to the total distance travelled in the solution of the CVRP with a homogeneous fleet with 30 -foot trucks.
For various instance sizes (50, 75 and 100 customers), the average decrease (in \%) in total distance with respect to a 30 -foot fleet solution is provided. The averages and standard deviations are calculated over 8 instances per problem class. As expected, for all instance sizes and in both problem classes, a decrease in total distance is observed when considering a 45-foot fleet instead of a 30foot fleet. An explanation for the decrease 
is that the capacity of a 45 -foot truck, in terms of number of pallets, is almost $50 \%$ higher than the capacity of a 30 -foot truck. As expected, the total distance has the lowest values for the FSM although the difference with a homogeneous fleet of 45 -foot trucks is rather small. This may be explained by the fact that on average over $85 \%$ of the routes in the solution of the FSM may be performed by 45 -foot trucks only.

Table 4

Decrease of the Total Distance Travelled (in Percentage) with Respect to the Homogeneous Fleet CVRP with 30-foot trucks - Scenario 1

\begin{tabular}{|l|c|c|c|c|c|c|}
\hline & \multicolumn{2}{|c|}{ 50 Customers } & \multicolumn{2}{c|}{ 75 Customers } & \multicolumn{2}{c|}{ 100 Customers } \\
\hline & 45 -foot & FSM & 45 -foot & FSM & 45-foot & FSM \\
\hline \multicolumn{7}{|c|}{ Problem Class 3 } \\
\hline Average & 18.78 & 19.53 & 20.03 & 20.32 & 21.68 & 21.44 \\
\hline Stand.Dev. & 1.02 & 0.91 & 0.94 & 0.63 & 0.78 & 1.08 \\
\hline \multicolumn{7}{|c|}{ Problem Class 4 } \\
\hline Average & 19.53 & 20.28 & 21.57 & 23.03 & 20.61 & 21.58 \\
\hline Stand.Dev & 1.78 & 1.29 & 2.49 & 1.91 & 1.24 & 1.23 \\
\hline
\end{tabular}

Table 5 presents the number of routes in the solution from the CVRP with sequence-based pallet loading and axle weight constraints for a vehicle fleet consisting of 30 -foot trucks, a fleet consisting of 45 -foot trucks and a heterogeneous vehicle fleet with 30 -foot and 45 -foot trucks. For all instance sizes, the average number of routes is lowest when only 45-foot trucks are considered. This implies that, although the weight capacity of a 45-foot truck is smaller than the weight capacity of a 30 -foot truck, in the given instances, more customers may be visited with a 45 -foot truck because of its larger capacity in terms of number of pallets. Because mostly 45-foot trucks are used in the FSM solutions, only a small difference exists in the number of routes between the fleet with 45-foot trucks and the heterogeneous fleet.

Table 5

Number of Routes in the Solution - Scenario 1

\begin{tabular}{|c|c|c|c|c|c|c|c|c|c|}
\hline & \multicolumn{3}{|c|}{50 Customers } & \multicolumn{3}{|c|}{75 Customers } & \multicolumn{3}{|c|}{100 Customers } \\
\hline & 30-foot & 45-foot & FSM & 30-foot & 45-foot & FSM & 30-foot & 45-foot & FSM \\
\hline \multicolumn{10}{|c|}{ Problem Class 3} \\
\hline Average & 13.54 & 9.43 & 9.85 & 19.88 & 14.15 & 14.39 & 27.15 & 19.14 & 19.60 \\
\hline Stand.Dev. & 0.49 & 0.44 & 0.40 & 0.37 & 0.35 & 0.38 & 0.48 & 0.23 & 0.47 \\
\hline \multicolumn{10}{|c|}{ Problem Class 4} \\
\hline Average & 19.89 & 13.80 & 14.53 & 30.91 & 21.68 & 22.40 & 40.38 & 28.81 & 29.69 \\
\hline Stand.Dev. & 1.65 & 1.20 & 1.45 & 1.87 & 1.39 & 1.42 & 2.87 & 1.90 & 2.11 \\
\hline
\end{tabular}

Table 6 presents the increase in distance travelled of the CVRP with axle weight constraints and sequence-based pallet loading compared to the equivalent CVRP without axle weight constraints. For each instance size (50, 75 and 100 customers), the increase in distance is provided for the model with a homogeneous fleet with 30 -foot trucks, a homogeneous fleet with 45-foot trucks and a heterogeneous fleet with 30 -foot and 
45-foot trucks. Results show that the effect of axle weight constraints on total distance travelled is highest when a homogeneous vehicle fleet of 45-foot trucks is considered with an average increase in distance of 6.72 $\%$ (over all problem sizes, over both problem classes). The increase for a homogeneous fleet of 30 -foot trucks is considerably smaller with an average of $2.33 \%$. The larger effect on 45 foot trucks is due to the fact that axle weight violations are more likely to occur on 45-foot trucks due to a higher tare weight and because more weight is applied on the coupling. As may be expected, the increase in distance, due to the integration of axle weight constraints, in the FSM is smaller than when only 45 -foot trucks are considered, but considerably higher than when only 30 -foot trucks are included. It may therefore be concluded that the vehicle fleet strongly influences the impact of the integration of axle weight constraints on the objective value.

Table 6

Increase in Total Distance (in Percentage) due to the Integration of Axle Weight Constraints - Scenario 1

\begin{tabular}{|l|c|c|c|c|c|c|c|c|c|}
\hline & \multicolumn{2}{l}{ 50 Customers } & \multicolumn{2}{l|}{ 75 Customers } & \multicolumn{2}{l|}{ 100 Customers } \\
\hline & 30 -foot & 45 -foot & FSM & 30-foot & 45 -foot & FSM & 30-foot & 45 -foot & FSM \\
\hline \multicolumn{8}{|c|}{ Problem Class 3 } \\
\hline Average & 0.80 & 4.09 & 3.07 & 2.13 & 5.10 & 4.44 & 2.72 & 6.28 & 6.06 \\
\hline Stand.Dev. & 0.59 & 1.00 & 0.90 & 0.64 & 1.05 & 1.00 & 0.50 & 0.65 & 1.01 \\
\hline \multicolumn{8}{|c|}{ Problem Class 4 } \\
\hline Average & 2.15 & 7.11 & 6.12 & 2.77 & 8.29 & 6.07 & 3.40 & 9.45 & 7.57 \\
\hline Stand.Dev. & 1.26 & 2.62 & 1.99 & 0.41 & 1.47 & 1.37 & 1.27 & 0.76 & 0.78 \\
\hline
\end{tabular}

\subsection{Results for the Objective 'Transport Cost Minimization'}

In this section, the results of the second scenario are discussed. The objective function aims to minimize total transport costs, as presented in Equation (3). Table 7 presents the percentage of routes in the final solutions of the FSM that are performed by 30 -foot trucks and by 45 -foot trucks with and without axle weight constraints. If a route leads to a feasible packing plan for both truck types, the route is assigned to a 30 -foot truck because the tare weight of a 30-foot truck is smaller than the tare weight of a 30 -foot truck and the weight is a determining factor of fuel costs. For this reason, the percentage of 45-foot trucks in the problem without axle weight constraints is smaller than in the first scenario, in which fuel costs are not considered. The average fleet composition of the problem with axle weight constraints is almost identical to the fleet composition of the problem without axle weight constraints.

\section{Table 7}

Routes Performed by 30-foot Trucks and 45-foot Trucks in the Final Solutions of the FSM in Percentage - Scenario 2

\begin{tabular}{|l|c|c|}
\hline & $\begin{array}{c}\mathbf{3 0} \mathbf{f t} \\
\text { Truck }\end{array}$ & $\begin{array}{c}\mathbf{4 5} \mathbf{f t} \\
\text { Truck }\end{array}$ \\
\hline $\begin{array}{l}\text { FSM without Axle } \\
\text { Weight Constraints }\end{array}$ & $16.13 \%$ & $83.87 \%$ \\
\hline $\begin{array}{l}\text { FSM with Axle } \\
\text { Weight Constraints }\end{array}$ & $16.69 \%$ & $83.31 \%$ \\
\hline
\end{tabular}


Table 8 presents the decrease of the solution cost (in percentage) from the CVRP with sequence-based pallet loading and axle weight constraints with a homogeneous vehicle fleet consisting of 45 -foot trucks and with a heterogeneous vehicle fleet with 30-foot and 45-foot trucks compared to the CVRP with a homogeneous fleet with 30foot trucks. For all instance sizes, a cost decrease is observed when considering a 45-foot fleet compared to a 30-foot fleet.
Note that the decrease in objective function value is smaller than in the first scenario. The reason for this may be that, in the current scenario, weight is a determining factor of fuel consumption and therefore has an impact on the solution cost. Since the tare weight of a 30-foot truck is smaller than the tare weight of a 45 -foot truck and the payload of 45-foot trucks is on average larger, the advantage of 45 -foot trucks in comparison to 30 -foot trucks is smaller in this scenario.

Table 8

Decrease of the Solution Cost (in Percentage) with Respect to the Homogeneous Fleet CVRP with 30foot Trucks - Scenario 2

\begin{tabular}{|l|c|c|c|c|c|c|}
\hline & \multicolumn{2}{|c|}{ 50 Customers } & 75 Customers & \multicolumn{2}{c|}{ 100 Customers } \\
\hline & 45 -foot & FSM & 45-foot & FSM & 45-foot & FSM \\
\hline \multicolumn{7}{|c|}{ Problem Class 3 } \\
\hline Average & 17.60 & 18.24 & 18.85 & 19.00 & 20.46 & 19.93 \\
\hline Stand.Dev. & 1.37 & 0.90 & 1.01 & 0.75 & 0.79 & 0.90 \\
\hline \multicolumn{7}{|c|}{ Problem Class 4 } \\
\hline Average & 17.93 & 19.42 & 21.28 & 22.50 & 20.38 & 21.42 \\
\hline Stand.Dev & 1.90 & 1.50 & 2.63 & 2.22 & 1.42 & 1.49 \\
\hline
\end{tabular}

The number of routes in the solution of the CVRP with sequence-based pallet loading and axle weight constraints for a vehicle fleet consisting of 30 -foot trucks, a fleet consisting of 45 -foot trucks and a heterogeneous vehicle fleet with 30-foot and 45-foot trucks are presented in Table 9. The results strongly resemble the results in Table 5 which indicates that the number of routes in the solutions is not affected by the different objective function.

\section{Table 9}

Number of Routes in the Solution - Scenario 2

\begin{tabular}{|l|c|c|c|c|c|c|c|c|c|c|}
\hline & \multicolumn{9}{c|}{ 50 Customers } & \multicolumn{3}{c|}{ 100 Customers } \\
\hline & 30-foot & 45 -foot & FSM & 30-foot & 45 -foot & FSM & 30-foot & 45-foot & FSM \\
\hline \multicolumn{8}{|c|}{ Problem Class 3 } \\
\hline Average & 13.68 & 9.73 & 9.93 & 19.89 & 14.19 & 14.45 & 27.15 & 19.31 & 19.71 \\
\hline Stand.Dev. & 0.51 & 0.41 & 0.30 & 0.38 & 0.29 & 0.36 & 0.69 & 0.31 & 0.60 \\
\hline \multicolumn{8}{|c|}{ Problem Class 4 } \\
\hline Average & 20.05 & 14.34 & 14.70 & 19.89 & 14.19 & 14.45 & 40.36 & 28.86 & 29.88 \\
\hline Stand.Dev. & 1.70 & 1.41 & 1.44 & 1.98 & 1.29 & 1.38 & 2.90 & 1.90 & 0.60 \\
\hline
\end{tabular}

Table 10 presents the cost increase of the CVRP with axle weight constraints and sequence-based pallet loading compared to the equivalent CVRP without axle weight constraints. For each instance size (50, 75 and 100 customers), the cost increase is provided 
for the model with a homogeneous fleet with 30 -foot trucks, a homogeneous fleet with 45foot trucks and a heterogeneous fleet with 30 -foot and 45-foot trucks. As in the first scenario, the increase in objective value of the integration of axle weight constraints is highest when a homogeneous vehicle fleet of 45-foot trucks is considered with an average cost increase of $2.99 \%$ (average over three problem size and over two problem classes). The cost increase of the integration of axle weight constraints is on average $2.28 \%$ for the FSM and $0.54 \%$ in case a homogeneous fleet of 30 -foot trucks is considered. Note that, for all fleet compositions, the increase in objective value is much smaller than in the first scenario. This effect can be explained as the fuel consumption is considered in the objective function; there appears a tendency to visit customers with heavy pallets early in the route. Therefore, heavy pallets are placed towards the rear of the vehicle. Since the weight capacity of the axles of the semitrailer is larger than the weight capacity of the axles of the tractor, the number of violations and the extent of the violation is therefore smaller. As a result, the difference in solution cost between the models with and without axle weight constraints is smaller too.

\section{Table 10}

Increase in Cost (in Percentage) due to the Integration of Axle Weight Constraints - Scenario 2

\begin{tabular}{|l|c|c|c|c|c|c|c|c|c|}
\hline & \multicolumn{3}{|c|}{ 50 Customers } & \multicolumn{3}{c|}{ 100 Customers } \\
\hline & 30 -foot & 45 -foot & FSM & 30-foot & 45 -foot & FSM & 30-foot & 45 -foot & FSM \\
\hline \multicolumn{8}{|c|}{ Problem Class 3 } \\
\hline Average & -0.09 & 2.08 & 1.57 & 0.10 & 1.53 & 1.23 & -0.55 & 1.64 & 2.54 \\
\hline Stand.Dev. & 0.28 & 1.46 & 0.70 & 0.76 & 0.97 & 0.84 & 0.74 & 0.71 & 1.01 \\
\hline \multicolumn{8}{|c|}{ Problem Class 4 } \\
\hline Average & 1.23 & 5.53 & 3.86 & 1.63 & 4.13 & 2.60 & 0.89 & 2.99 & 1.86 \\
\hline Stand.Dev. & 1.37 & 2.27 & 1.46 & 0.95 & 1.69 & 1.64 & 1.20 & 0.85 & 0.60 \\
\hline
\end{tabular}

\section{Conclusion and Future Research}

In real-life applications, the vehicle fleet of a transportation company is generally not homogeneous but consists of several vehicle types. This research considers the integration of a heterogeneous fleet in the CVRP with sequence-based pallet loading and axle weight constraints. As the number of vehicles in the heterogeneous fleet is unlimited the resulting problem is defined as a Fleet Size and Mix VRP with sequence-based pallet loading and axle weight constraints.

The effect of axle weight constraints for different fleet compositions on objective functions is compared. An Iterated Local Search heuristic is used to solve the problem. To measure the impact of the vehicle fleet on the integration of axle weight constraints in a VRP, a heterogeneous fleet with 30foot and 45-foot trucks is compared to a homogeneous fleet with 30 -foot trucks and a homogeneous fleet with 45 -foot trucks. Furthermore, two scenarios are analyzed for which the objective function is different. In the first scenario, the objective aims to minimize the total distance traveled while, in the second scenario, the objective aims to minimize the total transport costs.

The experimental results indicate that the effect of the axle weight constraints 
on the objective value is highest when a homogeneous fleet of 45 -foot trucks is considered. It can be explained because axle weight violations are more likely to occur in case axle weight constraints are ignored in 45-foot trucks since the capacity in terms of number of pallets is higher while the maximum weight of the load on the axles is smaller. Furthermore, the results indicate that, although the optimal composition of the fleet in the FSM mainly consists of 45-foot trucks, the effect of the integration of axle weight constraints on the objective value is smaller than, in the case, a homogeneous fleet of 45-foot trucks is considered. As expected, the overall objective value is lowest for the FSM with 30-foot and 45-foot trucks.

Based on these results, it may be concluded that the vehicle type, as well as the combination of vehicle types in the fleet, is of importance when calculating the impact of axle weight constraints on the objective function value. Besides, it is of interest to look at total transport costs, instead of total distance in the objective function, since the impact of the integration of axle weight constraints on the objective value is different for both objective functions. In case total transport costs are considered, the increase in objective value due to the integration of axle weight constraints is considerably smaller than when the objective is to minimize distance traveled.

Furthermore, results indicate that fleet size and mix decisions may be influenced by axle weight considerations. It may therefore be useful to consider axle weight constraints in long-term planning decisions concerning the acquisition of new vehicles. This may lead to a more diverse vehicle fleet with, for instance, vehicles with a smaller capacity in terms of number of pallets and a similar capacity in terms of total weight (e.g. 30-foot trucks instead of 45-foot trucks).

Future research could analyze the impact of axle weight constraints, in case other vehicle types are considered such as medium-duty trucks with different axle configurations. Furthermore, the effect of axle weight constraints on the FSM with sequence-based pallet loading and axle weight constraints with a fleet consisting of medium-duty and heavy-duty trucks may be analyzed. For this problem, fuel consumption models that do not only consider weight but also vehicle specific parameters such as average speed and acceleration may be used.

\section{Appendix A: Determination of the Center of Gravity}

In this appendix, a formula is developed to calculate the center of gravity of the pallets of a customer. Note that this development is valid for any vehicle type $k$, so the suffix $k$ is dropped in this Appendix. The calculation of the center of gravity is composed of two parts. The first part determines the starting point $\left(S_{j}\right)$ at which the first pallet of customer $j$ will be placed. This point depends on $l_{i j}$. If the number of pallets already in the truck is even, $S_{j}=l_{i j} / 2$. If the number is odd, $S_{j}=l_{i j} /$ $2-0.5$. The second part of the calculation of the center of gravity determines the distance between the center of gravity of the pallets of customer $j$ and $S$. This distance depends on the value of $l_{i j}, L_{j}$ and the capacity of the vehicle in terms of pallets, $L$.

In case $l_{i j}$ is even, the second part of the equation for the center of gravity is equal to $E_{j}$. $E_{j}$ corresponds to the center of gravity of the pallets of customer $j$ when the truck is empty on arrival $\left(l_{i j}=0\right) . E_{j}$ corresponds to equation A.1 or A.2, depending on whether 
$L_{i j}$ is respectively even or odd. The center of gravity of each pallet separately is summed up and divided by the number of pallets of customer $j\left(L_{j}\right)$.

If $L_{j}$ is even:

$E_{j}=\frac{\left(\operatorname{Max}\left[0,\left(L_{j}-1\right)\right]+\operatorname{Max}\left[0,\left(L_{j}-3\right)\right]+\cdots+\operatorname{Max}\left[0,\left(L_{j}-L-1\right)\right]\right)}{L_{j}}$

If $L_{j}$ is odd:

$E_{j}=\frac{\left(L_{j} / 2+\operatorname{Max}\left[0,\left(L_{j}-2\right)\right]+\operatorname{Max}\left[0,\left(L_{j}-4\right)\right]+\cdots+\operatorname{Max}\left[0,\left(L_{j}-L-2\right)\right]\right)}{L_{j}}$

In case $l_{i j}$ is odd, the second part of the equation for the center of gravity is equal to $O_{j} . O_{j}$ corresponds to the center of gravity of the pallets of customer $j$ when a single pallet is placed in the truck on arrival $\left(l_{i j}=\right.$ 1). $O_{j}$ corresponds to equation A.3 or A.4, depending on whether $L_{i j}$ is respectively even or odd. The calculation of $O_{j}$ is similar to the calculation of $E_{j}$. The last term in equation (A.3) is equal to the center of gravity of the first pallet of customer $j$ that is placed inside the truck. In equation (A.4), the last two terms make up the center of gravity of the first pallet of customer $j$ that is placed inside the truck.

If $L_{j}$ is even:

$E_{j}=\frac{\left(\left(L_{j}-1\right) / 2+\operatorname{Max}\left[0,\left(L_{j}-1\right)\right]+\operatorname{Max}\left[0,\left(L_{j}-3\right)\right]+\cdots+\operatorname{Max}\left[0,\left(L_{j}-L-1\right)+0.5\right]\right)}{L_{j}}$

If $L_{j}$ is odd:

$E_{j}=\frac{\left(L_{j}+\operatorname{Max}\left[0,\left(L_{j}-2\right)\right]+\operatorname{Max}\left[0,\left(L_{j}-4\right)\right]+\cdots+\operatorname{Max}\left[0,\left(L_{j}-L-2\right)-0.5\right]\right)}{L_{j}}$

Since the number of pallets of customer $j\left(L_{j}\right)$ and the vehicle capacity in terms of pallets $(L)$ known in advance, $O_{j}$ and $E_{j}$ can be treated as parameters or constants in the optimization model, formulated in Section 3. To integrate the equations (A.1), (A.2), (A.3) and (A.4) and the calculation of $S_{j}$ into a single formula to determine the center of gravity, parameter $P_{j}$ and variable $C_{i j}$ are created. $P_{j}=1$ if $L_{j}$ is even and $P_{j}=-1$ if $L_{j}$ is odd. Variable $C_{i j}$ is defined to keep track of the variable $l_{i j}$. When a vehicle travels from customer $i$ to $j, C_{i j}=1$ if $l_{i j}$ is even and $C_{i j}=-1$ if $l_{i j}$ is odd. When a vehicle does not travel from customer $i$ to $j, C_{i j}=0$.

The integrated formula of $C G$ is displayed in equation (A.5). Note that this is a linear equation.

$C G_{j}=\frac{\sum_{i \in V, i \neq j} l_{i j}}{2}-\frac{1}{4}\left(1-\sum_{i \in V, i \neq j} C_{i j}\right)+\frac{1}{2} O_{j}(1-$
$\left.-\sum_{i \in V, i \neq j} C_{i j}\right)+\frac{1}{2} E_{j}\left(1+\sum_{i \in V, i \neq j} C_{i j}\right), \forall j \in V$

The first two terms of (A.5) determine the starting point $S_{j}$ at which the first pallet of customer $j$ will be placed. If $l_{i j}$ is even, $C_{i j}=$ 1 and the second term vanishes. If $l_{i j}$ is odd, $C_{i j}=-1$ and the second term becomes -0.5 . The last two terms of (A.5) calculate the distance between the center of gravity of the pallets of customer $j$ and the front of the container, when no pallets or a single pallet are inside the truck. The distance is equal to the distance between the center of gravity of the pallets, and the starting point $S_{j}$. If $l_{i j}$ is even, $C_{i j}=1$ and the first term vanishes whole the second term becomes $E_{j}$. If $l_{i j}$ is odd, $C_{i j}$ $=-1$ and the first term becomes $O_{j}$ while the second term turns to zero.

\section{References}

Alonso, M.T.; Alvarez-Valdes, R.; Iori, M.; Parreño, F.; Tamarit, J. 2017. Mathematical models for multicontainer loading problems, Omega 66: 106-117.

Alonso, M.T.; Alvarez-Valdes, R.; Iori, M.; Parreno, F. 2019. Mathematical models for multi-container loading problems with practical constraints, Computers \& Industrial Engineering 127: 722-733. 
Alonso, M.T.; Alvarez-Valdes, R.; Parreno, F. 2020. A GRASP algorithm for multi-container loading problems with practical constraints, 4OR 18(1): 49-72.

Baldacci, R.; Battarra, M.; Vigo, D. 2008. Routing a Heterogeneous Fleet of Vehicles, The Vehicle Routing Problem: Latest Advances and New Challenges. Vol. 43 of the series Operations Research/Computer Science Interfaces, Springer US, Boston, 3-27.

Bräysy, O.; Porkka, P.; Dullaert, W.; Repoussis, P.; Tarantilis, C. 2009. A well-scalable metaheuristic for the fleet size and mix vehicle routing problem with time windows, Expert Systems with Applications 36(4): $8460-8475$.

Caceres-Cruz, J.; Arias, P.; Guimarans, D.; Riera, D.; Juan, A.A. 2014. Rich Vehicle Routing Problem: Survey, ACM Computing Surveys 47(2): 1-28.

Demir, E.; Bektaş, T.; Laporte, G. 2011. A comparative analysis of several vehicle emission models for road freight transportation, Transportation Research Part D: Transport and Environment 16(5): 347 - 357.

Golden, B.; Assad, A.; Levy, L.; Gheysens, F. 1984. The fleet size and mix vehicle routing problem, Computers \& Operations Research 11(1): $49-66$.

Hartl, R.F.; Hasle, G.; Janssens, G.K. 2006. Special issue on rich vehicle routing problems, Central European Journal of Operations Research 14(2): 103-104.

Hasle, G.; Kloster, O. 2007. Industrial vehicle routing. In Geometric Modelling, Numerical Simulation, and Optimization, G. Hasle, K.A. Lie, and E. Quak (Eds.), Springer, 397-435.

Hiermann, G.; Puchinger, J.; Ropke, S.; Hartl, R.F. 2016. The electric fleet size and mix vehicle routing problem with time windows and recharging stations, European Journal of Operational Research 252(3): 995-1018.
Iori, M.; Martello, S. 2010. Routing problems with loading constraints, TOP 18: 4-27.

Jacob, B.; Feypell-de La Beaumelle, V. 2010. Improving truck safety: potential of weigh-in-motion technology, IATSS (International Association of Traffic and Safety Science) Research 34(1): 9-15.

Koç, C.; Bektas, T.; Jabali, O.; Laporte, G. 2014. The fleet size and mix pollution-routing problem, Transportation Research Part B: Methodological 70: 239 - 254.

Koç, C.; Bektas, T.; Jabali, O.; Laporte, G. 2016. Thirty years of heterogeneous vehicle routing, European Journal of Operational Research 249(1): 1 - 21.

Kopfer, H.W.; Schönberger, J.; Kopfer, H. 2014. Reducing greenhouse gas emissions of a heterogeneous vehicle fleet, Flexible Services and Manufacturing Journal 26(1): 221-248.

Lim, A.; Ma, H.; Qiu, C.; Zhu, W. 2013. The single container loading problem with axle weight constraints, International Journal of Production Economics 144: 358-369.

Lourenço, H.; Martin, O.; Stützle, T. 2010. Iterated local search: Framework and applications. In: Handbook of Metaheuristics, Gendreau, M., Potvin, J. (Eds.), Vol. 146 of International Series in Operations Research \& Management Science. Springer, 363-397.

Männel, D.; Bortfeldt, A. 2016. A hybrid algorithm for the vehicle routing problem with pickup and delivery and three-dimensional loading constraints, European Journal of Operational Research, 254(3): 840-858.

Ostermeier, M.; Martins, S.; Amorim, P.; Hübner, A. 2018. Loading constraints for a multi-compartment vehicle routing problem, OR Spectrum 40(4): 997-1027.

Paquay, C.; Limbourg, S.; Schyns, M. 2018. A tailored two-phase constructive heuristic for the three- 
dimensional multiple bin size bin packing problem with transportation constraints, European Journal of Operational Research 267(1): 52-64.

Pollaris, H.; Braekers, K.; Caris, A.; Janssens, G.K.; Limbourg, S. 2015. Vehicle routing problems with loading constraints: State-of-the-art and future directions, OR Spectrum 37: 297-330.

Pollaris, H.; Braekers, K.; Caris, A.; Janssens, G.K.; Limbourg, S. 2016. Capacitated vehicle routing problem with sequence-based pallet loading and axle weight constraints, EURO Journal of Transportation and Logistics 5: 231-255.

Pollaris, H.; Braekers, K.; Caris, A.; Janssens, G.K.; Limbourg, S. 2017. Iterated local search for the capacitated vehicle routing problem with sequence-based pallet loading and axle weight constraints, Networks 69(3): 304-316.
Taillard, E.D. 1999. A heuristic column generation method for the heterogeneous fleet VRP, RAIRO Operations Research-Recherche Opérationnelle 33(1): 1-14.

UK Department for Transport. 2007. Effects of payload on the fuel consumption of trucks. Prepared by M. Coyle; IMISE Ltd. Available from Internet: <https://imise. co.uk/wp-content/uploads/2017/09/RR5-Effects-ofPayload-on-the-Fuel-Consumption-of-Trucks.pdf $>$.

Wei, L.; Zhang, Z.; Zhang, D.; Leung, S.C.H. 2018. A simulated annealing algorithm for the capacitated vehicle routing problem with two-dimensional constraints, European Journal of Operational Research 265(3): 843-859.

Xiao, Y.; Zhao, Q.; Kaku, I.; Xu, Y. 2012. Development of a fuel consumption optimization model for the capacitated vehicle routing problem, Computers \& Operations Research 39(7): 1419 - 1431. 